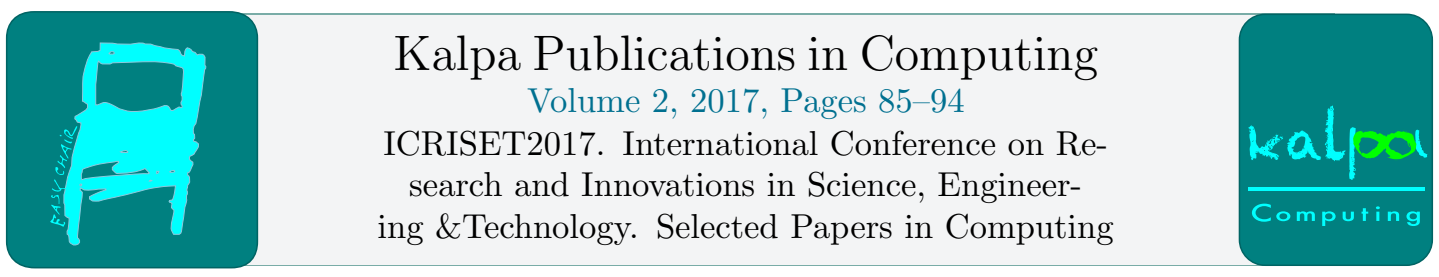

\title{
Optimal homotopy analysis solution of fingero-imbibition phenomenon in homogeneous porous medium with magnetic fluid effect
}

\author{
Dipak J. Prajapati ${ }^{1}$ and N. B. Desai ${ }^{2}$ \\ 1 Government Engineering College, Modasa-383315, Gujarat(INDIA) \\ djganit@gmail.com \\ 2 A. D. Patel Institute of Technology, New V. V. Nagar-388121, Gujarat(INDIA) \\ drnbdesai@yahoo.co.in
}

\begin{abstract}
The present paper discusses the fingero-imbibition phenomenon in a double phase displacement process through homogeneous porous medium with the involvement of a layer of magnetic fluid in the injected phase. This phenomenon has much importance in petroleum technology. The nonlinear partial differential equation governing this phenomenon with appropriate boundary conditions is solved by an optimal homotopy analysis method. The convergence of the solution is decided by minimizing discrete squared residual.
\end{abstract}

\section{Introduction}

When a porous medium filled with one phase (oil) is brought into the contact of another phase (water) which is preferentially wetting, then the spontaneous flow of the wetting phase into the medium and counter flow of the resident phase from the medium without any external force. This is known as imbibition phenomenon. Besides this if a phase (oil) contained in a porous medium is displaced by another phase of lesser viscosity, then instead of regular displacement of the whole front, protuberances may occur which shoot through the porous medium at relatively very great speed giving rise to the fingering phenomenon. This simultaneous occurrence of both phenomena was termed as fingero-imbibition by Verma.

Many researchers have discussed this problem from different viewpoints and solved by different methods: Mishra and Verma [14], Patel, Rabari and Bhathawala [19], Shah [26], Patel and Rabari [18],Shah and Verma [27], Parikh, Mehta and Pradhan [17], Verma and Rajput [31], Verma [30], Patel and Desai [20], Desai[4], Mehta [13] etc. In this paper, we have discussed the fingero-imbibition phenomenon through homogeneous porous medium with the involvement of a layer of magnetic fluid in the injected phase. In this work, the underlying assumptions are that the two phases are immiscible and the injected phase is less viscous as well as preferentially wetting. Also it is assumed that the magnetic field is directly proportional to the magnetic field intesity $H$ and the macroscopic behaviour of fingers is governed by a statistical treatment. An approximate analytical solution of the governing nonlinear partial differential equation with suitable boundary conditions has been obtained by optimal homotopy analysis method.

R. Buyya, R. Ranjan, S. Dutta Roy, M. Raval, M. Zaveri, H. Patel, A. Ganatra, D.G. Thakore, T.A. Desai, Z.H. Shah, N.M. Patel, M.E. Shimpi, R.B. Gandhi, J.M. Rathod, B.C. Goradiya, M.S. Holia and D.K. Patel (eds.), ICRISET2017 (Kalpa Publications in Computing, vol. 2), pp. 85-94 


\section{Formulation of the problem}

It is considered that a finite porous matrix with native phase $(n)$ is completely surrounded by an impermeable surface except for one end of the matrix which is labelled as the imbibition face $x=0$ and this end is exposed to an adjacent formation of injected phase $(i)$ which involves a thin layer of magnetic fluid. This arrangement gives rise to a displacement process in which the injection of the fluid $(i)$ is initiated by imbibition and the consequent displacement of native phase $(n)$ produces proturberances (fingers).

For the mathematical formulation, we assume that the Darcy's law is valid for the investigated flow system and the macroscopic behaviour of fingers is governed by a stastical treatment.[29] In this case, the saturation $\left(S_{i}(x, t)\right)$ of the injected phase $(i)$ is defined as the average cross-sectional area occupied by it at the level $(x)$ and time $(t)$ and thus the saturation of the displacing phase in the porous medium represents the average cross-sectional area occupied by the fingers. Let injected phase $(i)$ and native phase $(n)$ be two immiscible phases governed by Darcy's law and the velocities of water and oil be expressed as $[3,15]$

$$
\begin{aligned}
V_{i} & =-\frac{k_{i}}{\mu_{i}} K\left[\frac{\partial p_{i}}{\partial x}+\alpha H \frac{\partial H}{\partial x}\right] \\
V_{n} & =-\frac{k_{n}}{\mu_{n}} K \frac{\partial p_{n}}{\partial x}
\end{aligned}
$$

where

$$
\alpha=\mu_{0} \chi+\frac{16 \pi \mu_{0} \chi^{2} r^{3}}{9(l+2)^{3}}[27]
$$

$V_{i}$ and $V_{n}$ are the velocities of water and oil respectively, $K$ is the permeability of the homogeneous porous medium which is constant, $k_{i}$ and $k_{n}$ are the relative permeabilities of water and oil respectively, $\mu_{i}$ and $\mu_{n}$ are the constant viscosities of water and oil respectively, $p_{i}$ and $p_{n}$ are the pressures of water and oil respectively, $H$ is the magnetic field intensity, $\mu_{0}$ is permeability (magnetic) of free space, $l$ is the centre to centre distance of magnetic fluid particles, $r$ is the radius of magnetic fluid particles and $\chi$ is susceptibility.

The additional term $\alpha H \frac{\partial H}{\partial x}$ on the right hand side of (1) represents the additional pressure gradient exerted due to the presence of a layer of magnetic fluid in the displacing phase $(i)$.

Here we consider densities of both phases as constant. Hence the mass conservation laws for both phases of the mixture have the form [3]:

$$
\begin{array}{r}
P \frac{\partial S_{i}}{\partial t}+\frac{\partial V_{i}}{\partial x}=0 \\
P \frac{\partial S_{n}}{\partial t}+\frac{\partial V_{n}}{\partial x}=0
\end{array}
$$

where $P$ is the porosity of the medium regarded as constant.

In finger-imbibition, the velocities of injected and native phases are in opposite direction. According to Scheidegger [24]

$$
V_{i}=-V_{n}
$$


The capillary pressure $p_{c}$ is a function of the phase saturation. According to Scheidegger [24], it may be written as

$$
p_{c}\left(S_{i}\right)=p_{n}-p_{i}
$$

and

$$
p_{c}=-\beta S_{i}
$$

where $\beta$ is a constant.

For definiteness of the mathematical analysis, the relationship between phase saturation and relative permeability as given by Scheidegger and Johnson [25] is used here.

$$
k_{i}=S_{i} \quad \text { and } \quad k_{n}=S_{n}
$$

From the definition of phase saturation,

$$
S_{i}+S_{n}=1
$$

Using (1) and (2) in (6), we have

$$
\frac{k_{i}}{\mu_{i}} K\left[\frac{\partial p_{i}}{\partial x}+\alpha H \frac{\partial H}{\partial x}\right]+\frac{k_{n}}{\mu_{n}} K \frac{\partial p_{n}}{\partial x}=0
$$

Using (7) and simplyfying, we have

$$
\frac{\partial p_{i}}{\partial x}=-\frac{k_{i} \mu_{n} \alpha H \frac{\partial H}{\partial x}+k_{n} \mu_{i} \frac{\partial p_{c}}{\partial x}}{k_{i} \mu_{n}+k_{n} \mu_{i}}
$$

On substituting the value of (12) in (1) and simplyfying, we get

$$
V_{i}=K \frac{k_{i} k_{n}}{k_{i} \mu_{n}+k_{n} \mu_{i}}\left[\frac{\partial p_{c}}{\partial x}-\alpha H \frac{\partial H}{\partial x}\right]
$$

Also it is assumed that

$$
\frac{k_{i} k_{n}}{k_{i} \mu_{n}+k_{n} \mu_{i}} \approx \frac{k_{n}}{\mu_{n}}[24]
$$

Hence (13) reduces to

$$
V_{i}=K \frac{k_{n}}{\mu_{n}}\left[\frac{\partial p_{c}}{\partial x}-\alpha H \frac{\partial H}{\partial x}\right]
$$

Using (14) in (4), we get

$$
P \frac{\partial S_{i}}{\partial t}+\frac{\partial}{\partial x}\left[K \frac{k_{n}}{\mu_{n}}\left[\frac{\partial p_{c}}{\partial x}-\alpha H \frac{\partial H}{\partial x}\right]\right]=0
$$

Using (8), (9) and (10) into (15), we obtain

$$
P \frac{\partial S_{i}}{\partial t}-\frac{\beta K}{\mu_{n}} \frac{\partial}{\partial x}\left[\left(1-S_{i}\right) \frac{\partial S_{i}}{\partial x}\right]+\frac{K \alpha H}{\mu_{n}} \frac{\partial H}{\partial x} \frac{\partial S_{i}}{\partial x}-\frac{K \alpha\left(1-S_{i}\right)}{\mu_{n}}\left[H \frac{\partial^{2} H}{\partial x^{2}}+\left(\frac{\partial H}{\partial x}\right)^{2}\right]=0
$$


Here we consider the auxiliary magnetic field $H$ in the $x$-direction only,

$$
H=\frac{\lambda}{x^{n}}[27]
$$

where $\lambda$ is a constant parameter and $n$ is an integer. Using (17) for $n=-1$ in (16), we obtain

$$
P \frac{\partial S_{i}}{\partial t}-\frac{\beta K}{\mu_{n}} \frac{\partial}{\partial x}\left[\left(1-S_{i}\right) \frac{\partial S_{i}}{\partial x}\right]+\frac{K x \alpha \lambda^{2}}{\mu_{n}} \frac{\partial S_{i}}{\partial x}-\frac{K \alpha \lambda^{2}\left(1-S_{i}\right)}{\mu_{n}}=0
$$

Using dimensionless variables

$$
X=\frac{x}{L}, \quad T=\frac{\beta K t}{P \mu_{n} L^{2}},
$$

(18) reduces to

$$
\frac{\partial S_{i}}{\partial T}+\frac{\partial}{\partial X}\left[S_{i} \frac{\partial S_{i}}{\partial X}\right]+C \frac{\partial}{\partial X}\left[S_{i} X\right]-\frac{\partial^{2} S_{i}}{\partial x^{2}}-C=0
$$

where

$$
C=\frac{\alpha \lambda^{2} L^{2}}{\beta}
$$

Eq.(19) is desired nonlinear partial differential equation of motion for the flow of two immiscible phases in homogeneous medium with effect of magnetic fluid.

Let at the common interface, the saturation of injected water be linear function of time, that is

$$
S_{i}(0, T)=a T \quad \text { for } T>0
$$

where $a$ is a constant.

Since, it is assumed that the porous medium is completely surrounded by an impermeable surface except for one end, we consider

$$
\frac{\partial S_{i}}{\partial X}(1, T)=0 \quad \text { for } T>0
$$

We solve equation (19) together with boundary conditions (20) and (21) using optimal homotopy analysis method.

\section{Solution of the problem using Optimal Homotopy Anal- ysis Method}

We choose

$$
S_{i_{0}}(X, T)=a T+X e^{-X}
$$

as the initial approximation of $S_{i}(X, T)$ which satisfies boundary conditions (20) and (21).

Besides we choose the auxiliary linear operator as

$$
\mathcal{L}[\phi(X, T ; q)]=\frac{\partial^{2} \phi(X, T ; q)}{\partial X^{2}}+\frac{\partial \phi(X, T ; q)}{\partial X}
$$


with the property

$$
\mathcal{L}[f]=0 \text { when } f=0 .
$$

Furthermore, based on governing equation (19), we define a nonlinear operator as

$$
\begin{aligned}
\mathcal{N}[\phi(X, T ; q)] & =\frac{\partial \phi(X, T ; q)}{\partial T}+\phi(X, T ; q) \frac{\partial^{2} \phi(X, T ; q)}{\partial X^{2}}-\frac{\partial^{2} \phi(X, T ; q)}{\partial X^{2}}+\left\{\frac{\partial \phi(X, T ; q)}{\partial X}\right\}^{2} \\
& +C\{\phi(X, T ; q)-1\}+C X \frac{\partial \phi(X, T ; q)}{\partial X}
\end{aligned}
$$

Let $c_{0}$ denote a nonzero auxiliary parameter. According to Liao [8], the zeroth order deformation equation is

$$
(1-q) \mathcal{L}\left[\phi(X, T ; q)-S_{i_{0}}(X, T)\right]=c_{0} q H(X, T) \mathcal{N}[\phi(X, T ; q)]
$$

where $q \in[0,1]$ is the embedding parameter, $H(X, T)$ is nonzero auxiliary function and $\phi(X, T ; q)$ is an unknown function. Obviously, when $q=0$ and $q=1$, we have from (24) and $(26)$,

$$
\phi(X, T ; 0)=S_{i_{0}}(X, T)
$$

and

$$
\phi(X, T ; 1)=S_{i}(X, T)
$$

Therefore, $\phi(X, T ; q)$ varies from the initial approximation $S_{i_{0}}(X, T)$ to the solution $S_{i}(X, T)$ of the equation (19) as the embedding parameter $q$ increases from 0 to 1 . Obviously, $\phi(X, T ; q)$ is determined by the auxiliary linear operator $\mathcal{L}$, the initial guess $S_{i_{0}}(X, T)$ and the auxiliary parameter $c_{0}$. We have great freedom to select all of them. Assuming that all of them are so properly chosen that the Taylor series

$$
\phi(X, T ; q)=S_{i_{0}}(X, T)+\sum_{m=1}^{\infty} S_{i_{m}}(X, T) q^{m}
$$

exists and converges at $q=1$, we have the homotopy-series solution

$$
S_{i}(X, T)=S_{i_{0}}(X, T)+\sum_{m=1}^{\infty} S_{i_{m}}(X, T)
$$

where

$$
S_{i_{m}}(X, T)=\left.\frac{1}{m !} \frac{\partial^{m} \phi(X, T ; q)}{\partial q^{m}}\right|_{q=0}
$$

Differentiating the zeroth order deformation equation (26) $m$ times with respect to the embedding parameter $q$ and then dividing by $m$ ! and finally setting $q=0$, we have the so called high order deformation equation

$$
\mathcal{L}\left[S_{i_{m}}(X, T)-\chi_{m} S_{i_{m-1}}(X, T)\right]=c_{0} H(X, T) \mathcal{R}_{m}(X, T)
$$

subject to the boundary conditions

$$
S_{i_{m}}(0, T)=0, \quad \frac{\partial S_{i_{m}}}{\partial X}(1, T)=0, \quad m \geq 1
$$


where

$$
\mathcal{R}_{m}(X, T)=\left.\frac{1}{(m-1) !} \frac{\partial^{m-1} \mathcal{N}[\phi(X, T ; q)]}{\partial q^{m-1}}\right|_{q=0}
$$

and

$$
\chi_{m}= \begin{cases}0 & \text { if } m \leq 1 \\ 1 & \text { if } m>1\end{cases}
$$

It is very important to emphasize that (32) is linear for all $m \geq 1$. Thus we convert the original nonlinear problem into an infinite sequence of linear subproblems governed by (32). For simplicity, assume $H(X, T)=1$. The solution of the $m$ th order deformation equation (32) for $m \geq 1$ is

$$
S_{i_{m}}(X, T)=\chi_{m} S_{i_{m-1}}(X, T)+c_{0} \mathcal{L}^{-1}\left[\mathcal{R}_{m}(X, T)\right]+C_{1} X+C_{2}
$$

where $C_{1}$ and $C_{2}$ are constants or functions of $T$ and we determine them using boundary conditions (33). The equations (36) can be easily solved by symbolic computation software such as Mathematica. Hence the approximate analytical solution to the given nonlinear problem takes the following form:

$$
\begin{aligned}
S_{i}(X, T) & =a T+X e^{-X}-c_{0} \\
& -e c_{0}\left(1-e^{-X}\right)\left[a+a C T+\frac{C}{e}+\frac{1}{e}\left(\frac{1}{2}-\frac{C}{3}\right)-\frac{3}{e^{2}}-\frac{a T}{2 e}\right] \\
& +c_{0}\left[a X+a C T X-\left(X-\frac{X^{2}}{2}-\frac{C X^{3}}{3}\right) e^{-X}+\left(X^{2}+X+1\right) e^{-2 X}\right. \\
& \left.-\frac{a T\left(X^{2}-2 X\right) e^{-X}}{2}\right]+\cdots
\end{aligned}
$$

which represents the saturation of injected water $S_{i}(X, T)$ in homogeneous porous medium at distance $X$ and time $T$.

This solution expression contains the auxiliary parameter $c_{0}$ which is called the convergencecontrol parameter and is employed to control the convergence of the solution.

According to Liao[11], the discrete squared residual at the $m$ th order of approximation is

$$
E_{m}=\frac{1}{(M+1)(N+1)} \sum_{i=0}^{M} \sum_{j=0}^{N}\left\{\mathcal{N}\left[\sum_{n=0}^{m} S_{i_{n}}\left(\frac{i}{M}, \frac{j}{N}\right)\right]\right\}^{2}
$$

The value of $c_{0}$ can be optimally identified from the condition

$$
\frac{d E_{m}\left(c_{0}\right)}{d c_{0}}=0
$$

This optimization approach for obtaining the parameter $c_{0}$ has been applied recently to a number of problems for nonlinear ordinary and partial differential equations $[9,10,16,2,23$, $21,22,5,6,1,12,32,28,7]$. Fig. 1 shows the curve of discrete squared residual at the 5 th order of approximation $E_{5}$ versus $c_{0}$. 


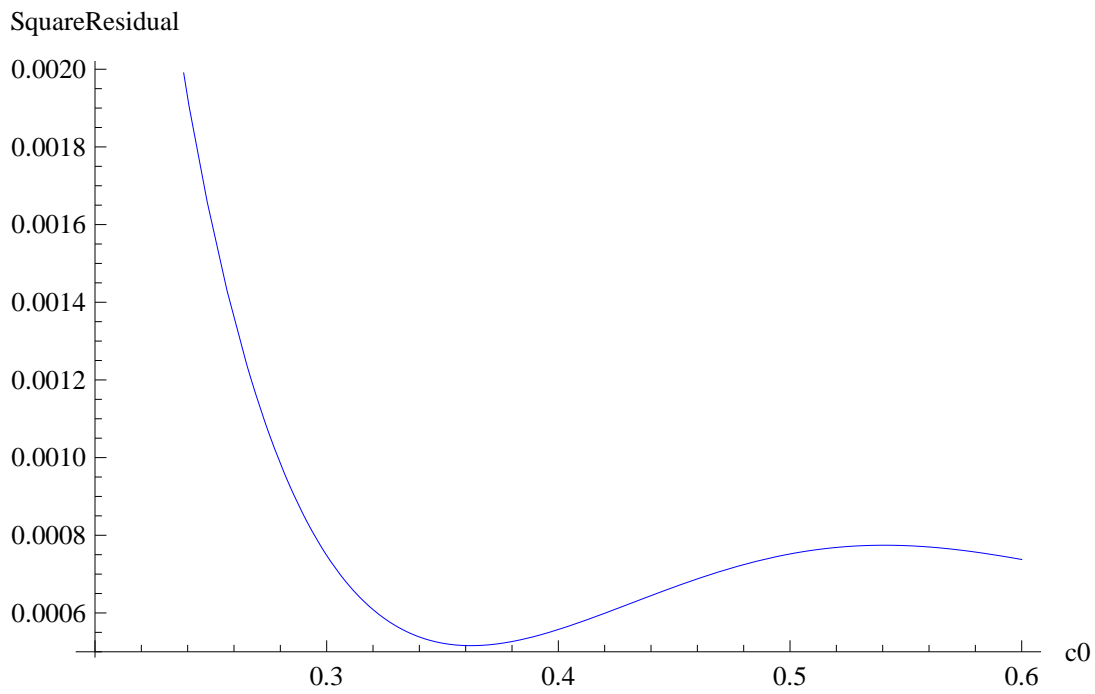

Fig. 1: $E_{5}$ versus $c_{0}$.

Using Mathematica, we find that square residual $E_{5}$ has its minimum value $5.15788 \times 10^{-4}$ at $c_{0}=0.362293$ for $M=N=50$ which can be seen in Fig. 1 also.

\section{Numerical and Graphical Representation}

We obtain the numerical and graphical representations of the solution $S_{i}(X, T)$ up to 5 th order approximation using $c_{0}=0.362293$. Also we use the following values of parameters:

$$
\begin{gathered}
L=1.0 \mathrm{~m}, \beta=6895 \mathrm{~N} / \mathrm{m}^{2}, \mu_{0}=4 \pi \times 10^{-7} \mathrm{H} / \mathrm{m}, \\
\chi=0.2805031, \lambda=1.0, r=1.03281 \times 10^{-9} \mathrm{~m}, l=20 \times 10^{-9} \mathrm{~m}, \\
C=5.10515 \times 10^{-11}, a=0.001, \alpha=3.52 \times 10^{-7} \mathrm{H} / \mathrm{m} .
\end{gathered}
$$

Numerical values of the saturation of injected water obtained by the optimization approach up to 5th order approximation are given in Table 1. Fig. 2 represents the graph of saturation of injected water, $S_{i}(X, T)$, versus distance $X$ for fixed values of time $T=200,250,300, \cdots, 600$. Fig. 3 represents the graph of saturation of injected water, $S_{i}(X, T)$, versus time $T$ for fixed values of distance $X=0.1,0.2, \cdots, 1$. We use numerical values of Table 1 for Fig. 2 and Fig. 3 . 
Table 1: Numerical values of the saturation of injected water.

\begin{tabular}{|c|c|c|c|c|c|c|c|c|c|c|}
\hline$T$ & $X=0.1$ & $X=0.2$ & $X=0.3$ & $X=0.4$ & $X=0.5$ & $X=0.6$ & $X=0.7$ & $X=0.8$ & $X=0.9$ & $X=1$ \\
\hline 200 & 0.201311 & 0.202704 & 0.204440 & 0.206539 & 0.208856 & 0.211174 & 0.213261 & 0.214916 & 0.215982 & 0.216353 \\
250 & 0.253108 & 0.256342 & 0.259864 & 0.263593 & 0.267314 & 0.270764 & 0.273700 & 0.275926 & 0.277309 & 0.277780 \\
300 & 0.305367 & 0.310882 & 0.316581 & 0.322272 & 0.327661 & 0.332443 & 0.336364 & 0.339248 & 0.340995 & 0.341575 \\
350 & 0.358148 & 0.366436 & 0.374748 & 0.382767 & 0.390113 & 0.396443 & 0.401502 & 0.405138 & 0.407297 & 0.408002 \\
400 & 0.411515 & 0.423124 & 0.434531 & 0.445279 & 0.454900 & 0.463013 & 0.469372 & 0.473863 & 0.476488 & 0.477333 \\
450 & 0.465536 & 0.481072 & 0.496106 & 0.510022 & 0.522263 & 0.532416 & 0.540252 & 0.545708 & 0.548855 & 0.549857 \\
500 & 0.520283 & 0.540416 & 0.559658 & 0.577223 & 0.595459 & 0.604929 & 0.614432 & 0.620971 & 0.624701 & 0.625877 \\
550 & 0.575833 & 0.601298 & 0.625383 & 0.647119 & 0.665756 & 0.680842 & 0.692218 & 0.699967 & 0.704345 & 0.705714 \\
600 & 0.632268 & 0.663868 & 0.693490 & 0.719962 & 0.742439 & 0.760462 & 0.773930 & 0.783025 & 0.788121 & 0.789703 \\
\hline
\end{tabular}

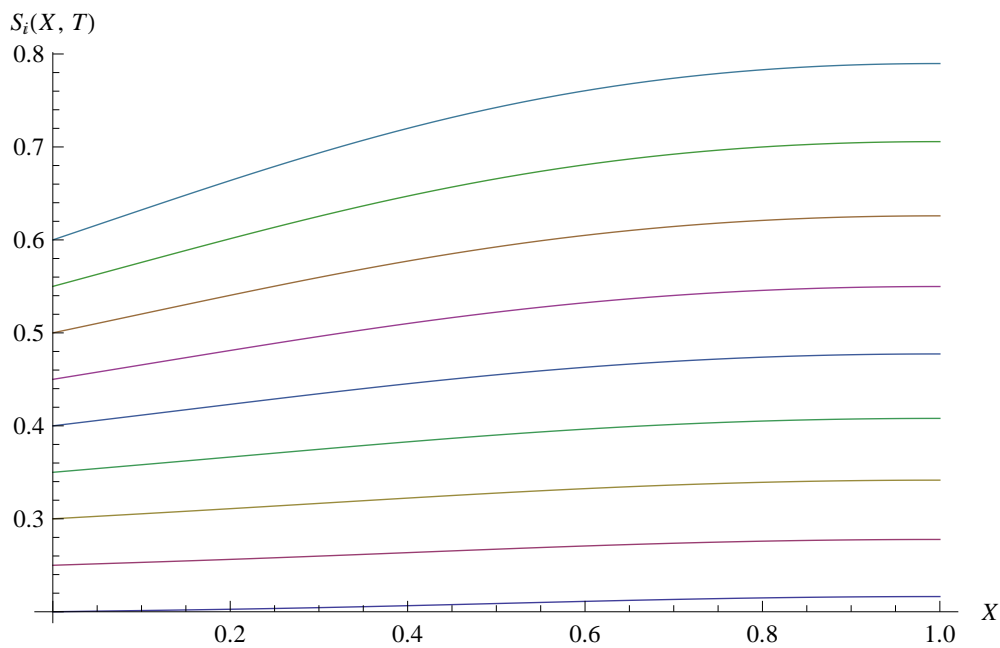

Fig. 2: $S_{i}(X, T)$ versus $X$ for fixed values of times

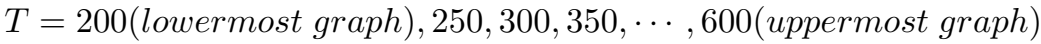

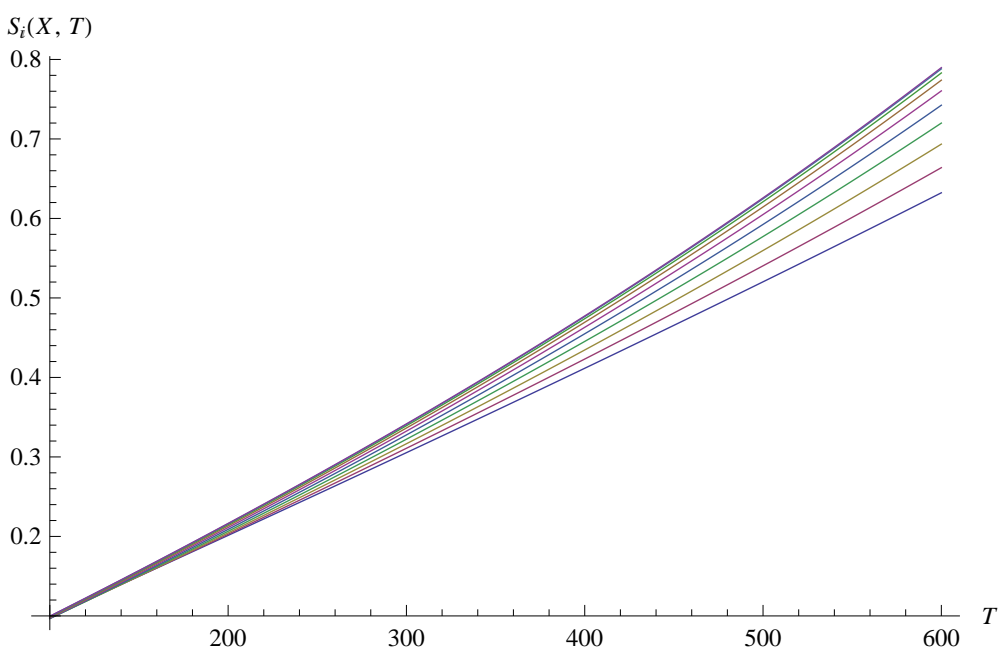

Fig. 3: $S_{i}(X, T)$ versus $T$ for fixed values of distance $X=0.1$ (lowermost graph) $, 0.2, \cdots, 1$ (uppermost graph) 


\section{Conclusion}

In this paper we have used the optimal homotopy analysis method to obtain the approximate analytical solution for the fingero-imbibition phenomenon in homogeneous porous medium with magnetic fluid effect. The optimal homotopy analysis method provides us a simple way to adjust and control the convergence of the series solution by choosing proper value of auxiliary parameter. From Fig. 2, we can see that the saturation of injected water increases when the distance increases for fixed value of time. Also the saturation of water increases when time increases for fixed value of distance which can be observed in Fig. 3. This is consistent with real phenomenon.

\section{References}

[1] S. Abbasbandy and M. Jalili. Determination of optimal convergence-control parameter value in homotopy analysis method. Numer Algor., 2013.

[2] M. Baxter, A. Robert, V. Gorder, and K. Vajravelu. On the choice of auxiliary linear operator in the optimal homotopy analysis of the cahn-hilliard initial value problem. Numer Algor.,66(2), 269-298., 2014.

[3] J. Bear. Dynamics of fluids in porous media. American Elsevier Publishing Company, Inc., New York., 1972.

[4] N. B. Desai. The study of problems arises in single phase and multiphase flow through porous media. Ph.D. Thesis, South Gujarat University, Surat, India., 2002.

[5] T. Fan and X. You. Optimal homotopy analysis method for nonlinear differential equations in the boundary layer. Numer Algor.,62, 337-354., 2013.

[6] H.N. Hassan and M.S. Semary. Analytic approximate solution for the bratu's problem by optimal homotopy analysis method. International Scientific Publications and Consulting Services., 2013.

[7] J. Izadian and M. MohammadzadeAttar. Numerical solution of deformation equations in homotopy analysis method. Applied Mathematical Sciences.,6(8), 357-367., 2012.

[8] S. J. Liao. Beyond perturbation: Introduction to the homotopy analysis method. Chapman and Hall/CRC Press, Boca Raton., 2003.

[9] S. J. Liao. An optimal homotopy analysis approach for strongly nonlinear differential equations. Commun. Nonlinear Sci. Numer. Simul., 15, 2003-2016., 2010.

[10] S. J. Liao. Advances in the homotopy analysis method. World Scientific., 2013.

[11] S.J. Liao. Homotopy analysis method in nonlinear differential equations. Higher Education Press, Beijing and Springer-Verlag Berlin Heidelberg., 2012.

[12] K. Mallory and V. Gorder. Optimal homotopy analysis and control of error for solutions to the non-local whitham equation. Numer Algor., 2013.

[13] M. N. Mehta. Asymptotic expansions of fluid flow through porous media. Ph.D. Thesis, South Gujarat University, Surat, India., 1977.

[14] A.K. Mishra and A. P. Verma. Imbibition in the flow of two immiscible fluids with magnetic field. Physics of Fluids, 17., 1974.

[15] M. Muskat. The flow of homogeneous fluids through porous media. First edition, McGraw-Hill Book Company, Inc., New York and London., 1937.

[16] J.S. Nadjafi and J.S. Jafari. Comparison of liao's optimal ham and niu's one-step optimal ham for solving integro-differential equations. Journal of Applied Mathematics and Bioinformatics.,1(2), 85-98., 2011.

[17] A.K Parikh, M. N. Mehta, and V.H. Pradhan. Mathematical modeling and analysis of fingeroimbibition phenomenon in homogeneous porous medium with magnetic field effect in vertical 
downward direction. Ijltemas, 3(10)., 2014.

[18] A.V. Patel, N.S. Rabari, and Bhathawala P.H. Numerical solution of imbibition phenomenon in a homogeneous medium with magnetic fluid. IOSR Journal of Mathematics, 11(4)., 2015.

[19] A.V. Patel, N.S. Rabari, and Bhathawala P.H. A mathematical model for fingero-imbibition phenomenon in a cracked porous media with magnetic fluid. International Journal of Advanced Technology in Engineering and Science, 4(4)., 2016.

[20] M. A. Patel and N. B. Desai. Homotopy analysis solution of countercurrent imbibition phenomenon in inclined homogeneous porous medium. Global Journal of Pure and Applied Mathematics, 12(1), 1035-1052., 2016.

[21] D. J. Prajapati and N. B. Desai. The solution of immiscible fluid flow by means of optimal homotopy analysis method. International Journal of Computer and Mathematical Sciences.,4(8)., 2015.

[22] D. J. Prajapati and N. B. Desai. Application of the basic optimal homotopy analysis method to fingering phenomenon. Global Journal of Pure and Applied Mathematics.,12(3), 2011-2022., 2016.

[23] J. Saeidian and S. Javadi. Some notes on the convergence control parameter in the framework of the homotopy analysis method. Journal of Mathematics and Computer Science.,9, 103-110., 2014.

[24] A. E. Scheidegger. The physics of flow through porous media. Revised edition, University of Toronto Press, Toronto., 1960.

[25] A. E. Scheidegger and E. F. Johnson. The statistically behaviour of instabilities in displacement process in porous media. Canadian Journal of Physics, 39(2), 326-334., 1961.

[26] R.C. Shah. Magnetic fluid effect on fingero-imbibition phenomenon in secondary recovery process in transport phenomenon. Indian Journal of Chemical Technology, 8, 344-347., 2001.

[27] R.C. Shah and A.P. Verma. Fingero-imbibition phenomenon through porous media with magnetic fluid. Indian Journal of Engineering and Material Sciences, 2., 1998.

[28] K. Vajravelu and R. A. Van Gorder. Nonlinear flow phenomena and homotopy analysis: Fluid flow and heat transfer. Higher Education Press, Beijing and Springer-Verlag Berlin Heidelberg., 2012.

[29] A. P. Verma. Statistical behaviour of fingering in a displacement process in heterogeneous porous medium with capillary pressure. Canadian Journal of Physics, 47(3), 319-324., 1969.

[30] A. P. Verma. Fingero-imbibition in artificial replenishment ground water through cracked porous medium. Water Resources Research, 6(3), 906-911., 1970.

[31] A.P. Verma and A.K. Rajput. Instabilities in displacement process through porous media with magnetic fluid. Journal of Magnetism and Magnetic Materials, 65, 330-334., 1987.

[32] K. Yabushita, M. Yamashita, and K. Tsuboi. An analytic solution of projectile motion with the quadratic resistance law using the homotopy analysis method. J.Phys. A-Math.Theor., 40, 8403-8416., 2007. 